Leading Article

\title{
The problem of viral heart disease. How often do we miss it?
}

\author{
E.G.J. Olsen
}

National Heart Hospital, London W1M 8BA, UK.

Myocarditis is now defined as the presence of inflammatory cells in the myocardium with evidence of fraying of adjacent myocardial fibres but without sequential fibre necrosis (as seen in ischaemic heart disease) (Olsen, 1981). It is one of the most difficult diagnoses to make both clinically and morphologically. One has only to trace the historical background of this discase to be aware of the difficulty that has beset myocarditis. Proof of an inflammatory process in the myocardium was often not present or sought, and ever since the introduction of the term by Sobernheim in 1837 up to about the 1930s the term was indiscriminately applied. Chronic myocarditis became a favourite diagnosis and included such conditions as hypertension (Olsen, 1983). Even the work of Herrick, which clearly established a vascular cause for myocardial damage and permitted separation from myocarditis due to an inflammatory process, was not heeded (Olsen, 1983). Dr Paul White and Sir Thomas Lewis drew attention to the misuse of the term and whilst not denying the existence of myocarditis stressed the overdiagnosis of this condition. This resulted in overcaution by clinicians and pathologists alike in making such a diagnosis thus resulting in an under-estimate of the incidence.

Many authorities have reported an incidence of myocarditis post mortem varying between $3.5 \%$ to 9\% (Olsen, 1980). Often, however, the inflammatory infiltrate may have disappeared and all that is found is non-specific interstitial fibrous tissue together with foci of fibrous replacement. From a clinical standpoint the diagnosis can be extremely difficult. Signs and symptoms are often non-specific and include chest pain, fever, cardiomegaly, flu-like symptoms, pulmonary oedema and lymphadenopathy (Smith, 1970).

Electrocardiographically ischaemic heart disease, and even myocardial infarction may be mimicked. This aspect of myocarditis is highlighted in a paper by O'Neill et al. in this issue where in a group of 12 patients a diagnosis of acute myocardial infarction was made. After examination, which included coron-

E.G.J. Olsen, M.D., F.R.C.Path., F.A.C.C.

Received: 5 December 1984 ary arteriography and viral serology, evidence of recent Coxsackie $B$ infection was evident and myocarditis in at least half the patients was thought to be the likely diagnosis. In these patients coronary arteriography showed no sigificant abnormal changes. This paper is therefore a timely reminder that in cases with 'myocardial infarction' and normal coronary arteries, virological studies should be undertaken to help to define the true underlying pathology. The symptomatology of myocardial infarction is well defined and it might be deemed unnecessary to subject such patients to coronary arteriography. Furthermore, how often are virological studies undertaken in patients with myocardial infarction?

It is therefore likely that myocarditis is missed in a substantial number of cases, and this supposition has been shown to be well founded since the introduction of endomyocardial biopsy examination on tissue obtained by bioptome. In a group of 1,156 patients clinically suspected to suffer from dilated cardiomyopathy - in which angina-like pain is not infrequently experienced-myocarditis has been found in 288 patients on morphological examination in these small tissue samples? In a study of 12 patients with virological evidence in 6 , and morphological evidence of active myocarditis in 11 patients, treatment with prednisolone and azathioprine in 8 patients and prednisolone alone in one patient has shown encouraging clinical responses in the majority of these patients. There was no doubt that virological investigation together with myocardial histology were complementary in the diagnosis of acute myocarditis (Daly et al., 1984).

This study also showed that myocarditis may be present without virological evidence whilst on the other hand positive virological serology was not necessarily indicative of myocarditis. This was also substantiated in two other studies (Olsen, 1983; Richardson et al., 1983; Cambridge et al., 1979). In one, in collaboration with the Department of Cardiology at King's College Hospital, of 74 patients with suspected dilated cardiomyopathy, 22 showed active myocarditis $45 \%$ of whom had positive serology (Olsen, 1983). These studies have shown that en- 
domyocardial biopsy enables assessment of a truer incidence of myocarditis which, without tissue examination, might otherwise well have been missed. It also eliminates those patients in whom myocarditis is clinically suspected and who, even in the presence of positive virological serology, may not show myocarditis. This was the case in another group of 214 patients in whom the clinically suspected diagnosis was myocarditis, confirmed in only half of them.

It could be argued that the small tissue samples obtained by biopsy may not be a true reflection of the rest of the myocardium and false negative results may be obtained. This aspect has been investigated by morphological studies and it has been shown that if 5 or more samples from the same ventricle are taken the coefficient of variance becomes acceptably low (just over 7) indicating good representation of the myocardium in these tissue samples (Baandrup et al., 1982). The technique of obtaining endomyocardial biopsies, though safe in experienced hands, is, nevertheless, invasive and as far as patients with myocarditis are concerned may not be acceptable when the disease is relatively mild or self-limiting (O'Neill et al., 1985).

Unfortunately, correlation between clinical symptomatology and intensity of the inflammatory infiltrate does not always exist and it would therefore be unwise to assume mild myocardial involvement on clinical grounds alone. Similarly the problem as to whether the condition is self-limiting has so far not been firmly established. To this end a multi-centre study between the United States of America and Europe has been set up to ascertain whether indeed self-limitation of the disease process occurs.

So far the remarks have concentrated on myocarditis due to Coxsackie B virus infection. It should be remembered that any virus can affect the heart. A fatal $\stackrel{\varnothing}{\AA}$ case of myocarditis due to varicella is also reported in $C$ this issue (Coppack et al., 1985. In this patient the clinical picture was dominated by neurological manifestations, and myocarditis was established only post mortem. The myocarditic process was not striking and was similar to changes observed in patients with dilated cardiomyopathy in whom myocarditis had been found. As the inflammatory infiltrate is often non-specific by the time endomyocardial biopsy (or $\vec{\circ}$ post mortem) is performed, it is helpful to undertake virological analysis to ascertain the type of virus. This paper underlines one of the many problems that exist. However vigilant the clinician is, myocarditis may be covert, being overshadowed by associated or complicating factors such as, in this case, by neurological manifestations. Other problems include the lack of $\neg$ universally accepted clinical criteria and criteria for histological interpretation.

Nevertheless despite these obvious shortcomings biopsy examination has greatly facilitated a more accurate assessment of the incidence of myocarditis. Whereas we may still only be dealing with the tip of an iceberg as far as the true incidence of myocarditis is concerned, non-invasive and invasive techniques aro now available to ensure a more accurate incidences which, without examination of tissue obtained b biopsy, would not be possible.

marson, A. \& Olsen, E.G.J. (eds), p. 122, A.B. Hassle: Molndal, Sweden.

OLSEN, E.G.J. (1983). Myocarditis - a case of mistaken identity? British Heart Journal, 50, 303.

O'NEILL, D., MCARTHUR, J.D., KENNEDY, J.A. \& CLEMENTS, G. (1985). Myocardial infarction and the normal arteriogram - possible role of viral myocarditis. Postgraduate Medical Journal, 61, 485.

RICHARDSON, P.J., MORGAN-CAPNER, P., DALY, K., MCSORLEY, C. \& OLSEN, E.G.J. (1983). Endomyocardial biopsy and viral heart disease. Verhandlung der Deutschen Gesellschaft für Herz und Kreislaufforschung, 49, 141.

SMITH, W.G. (1970). Coxsackie B Myopericarditis in adults. American Heart Journal, 80, 34.

SOBERNHEIM, J.F. (1837). Praktische Diagnostik der inbiopsy and viral heart disease. Verhandlung der Deutschen Gesellschaft für Herz und Kreislaufforschung, 49, 141.

SMITH, W.G. (1970). Coxsackie B Myopericarditis in adults. American Heart Journal, 80, 34.

SOBERNHEIM, J.F. (1837). Praktische Diagnostik der inneren Krankheiten mit vorzueglicher. In Ruecksicht auf pathologische Anatomie, p. 117. Hirschwald: Berlin.

OLSEN, E.G.J. (1981). Panel discussion. In Congestive cardiomyopathy, Kiruna, Sweden, 1980, Goodwin, J.F. Hjal- 